\title{
The Minimum Shared Edges Problem on Grid-like Graphs
}

\author{
Till Fluschnik ${ }^{* 1}$, Meike Hatzel ${ }^{1}$, Steffen Härtlein ${ }^{1}$, \\ Hendrik Molter ${ }^{\dagger}$, and Henning Seidler ${ }^{1}$ \\ ${ }^{1}$ Institut für Softwaretechnik und Theoretische Informatik, TU Berlin, Germany, \\ \{till.fluschnik, meike.hatzel, hendrik.molter\}@tu-berlin.de, \\ \{haertlein, henning.seidler\}@campus.tu-berlin.de
}

\begin{abstract}
We study the NP-hard Minimum Shared Edges (MSE) problem on graphs: decide whether it is possible to route $p$ paths from a start vertex to a target vertex in a given graph while using at most $k$ edges more than once. We show that MSE can be decided on bounded (i.e. finite) grids in linear time when both dimensions are either small or large compared to the number $p$ of paths. On the contrary, we show that MSE remains NP-hard on subgraphs of bounded grids.

Finally, we study MSE from a parametrised complexity point of view. It is known that MSE is fixed-parameter tractable with respect to the number $p$ of paths. We show that, under standard complexity-theoretical assumptions, the problem parametrised by the combined parameter $k, p$, maximum degree, diameter, and treewidth does not admit a polynomialsize problem kernel, even when restricted to planar graphs.
\end{abstract}

\section{Introduction}

Routing in street-like networks is a frequent task. Graphs modelling street networks are often (almost) planar, that is, they can be drawn in the plane with (almost) no edge crossings. As a special case, a graph modelling the street network in Manhattan is similar to a grid graph. We study the following problem, originally introduced by Omran et al. [15], from a computational (parametrised) complexity perspective on planar and grid-like graphs:

\section{Minimum Shared Edges (MSE)}

Input: $\quad$ An undirected graph $G=(V, E)$, two distinct vertices $s, t \in V$, and two integers $k, p \in \mathbb{N}$.

Question: Are there $p$ paths from $s$ to $t$ in $G$ such that at most $k$ edges appear in more than one of the $p$ paths?

Note that Omran et al. [15] originally defined the problem on directed graphs (we refer to this as Directed Minimum Shared Edges or DMSE). While Omran

\footnotetext{
*Supported by the DFG, project DAMM (NI 369/13-2).

†Partially supported by the DFG, project DAPA (NI 369/12).
} 
et al. motivate MSE by applications in security management, the problem can further appear in the following scenario. A network company wants to upgrade their network since it still uses old copper cables. To improve the throughput, some of these cables shall be replaced by modern optical fibre cables. The network routes information from a source location to a target location and the company wants to achieve a certain minimal throughput. Since digging up the conduits for the cables is much more expensive than the actual cables, we can neglect the cost of the cables and upgrade them to arbitrary bandwidth, because once open, we can lay as many cables as necessary into a conduit. The company wants to find the minimum number of conduits that have to be dug up in order to achieve the desired bandwidth.

Related Work. Omran et al. [15] showed that DMSE is NP-complete on directed acyclic graphs. The problems MSE and DMSE were both shown to be NP-complete even if the input graph is planar [8]. Moreover, MSE is solvable in linear time on unbounded (i.e. infinite) grid graphs [6]. DMSE is $\lfloor k / 2\rfloor$-approximable [3], but there is no polynomial-time approximation of factor $2^{(\log (n))^{1-\epsilon}}$ for any $\epsilon>0$ unless NP $\subseteq \operatorname{DTIME}\left(n^{\text {polylog }(n)}\right)[15]$.

Analysing its parametrised complexity, Fluschnik et al. [7] showed that MSE is fixed-parameter tractable when parametrised by the number $p$ of paths but does not admit a polynomial-size problem kernel unless NP $\subseteq$ coNP/poly, MSE is $\mathrm{W}[1]$-hard when parametrised by $\mathrm{tw}+k$, where tw denotes the treewidth of the input graph, and W[2]-hard when parametrised by the number $k$ of shared edges. Furthermore, MSE is solvable in polynomial time on graphs of bounded treewidth $[2,18]$.

Our Contribution. We give both positive and negative results for MSE on grid-like graphs. On the positive side, we show that if the dimensions of the grid are smaller than the number $p$ of paths, then MSE is trivially decidable, and if the dimensions of the grid are at least the number $p$ of paths, then we provide an arithmetic criterion to decide MSE in linear-time (Section 3.1). On the negative side, we prove that the situation changes when subgraphs of bounded grids (which we refer to as holey grids) are considered, that is, we prove that MSE on subgraphs of bounded grids is NP-hard (Section 3.2). Similarly, we prove that DMSE is NP-hard for acyclic subgraphs of directed bounded grids (Section 3.3). Our NP-hardness results improve upon the known hardness results [8] as the graphs we consider are more restricted. Moreover, we show that MSE parametrised by $k+p+\Delta+\operatorname{diam}+\mathrm{tw}$, where $\Delta$ and diam denote the maximum degree and diameter, respectively, does not admit a polynomial-size problem kernel, unless NP $\subseteq$ coNP/poly, even on planar graphs (Section 4), improving an existing kernelization lower bound [7].

\section{Preliminaries}

We use basic notation from graph theory and parametrised complexity. We denote by $\mathbb{N}$ the natural numbers containing zero.

Graph Theory. Unless stated otherwise, we assume that all graphs are finite, undirected, simple and without self-loops. We refer with $V(G)$ and $E(G)$ to the 
vertex set and edge set, respectively, of a graph $G$. An edge set $P \subseteq E$ is called a path if we have $P=\left\{\left\{v_{i-1}, v_{i}\right\} \mid 0<i \leq n\right\}$ for some pairwise distinct vertices $v_{0}, \ldots, v_{n}$. In this case we say $P$ is a $v_{0}-v_{n}$-path of length $n$. The distance $\operatorname{dist}_{G}(u, v)$ between two vertices $u, v \in V(G)$ is defined as the length of a shortest $u$-v-path (we set $\operatorname{dist}_{G}(u, v)=\infty$ if there is no $u$-v path in $G$ ).

Grids. For $n, m \in \mathbb{N}$, let $G_{n \times m}$ be the (bounded) $n \times m$-grid, that is, the undirected graph $(V, E)$ with the set of vertices $V:=\{(x, y) \in \mathbb{N} \times \mathbb{N} \mid x<n, y<m\}$ and the set of edges $E:=\{\{(v, w),(x, y)\}|| v-x|+| w-y \mid=1\}$.

The coordinates of a vertex are denoted by $v:=\left(v_{x}, v_{y}\right)$. We call the vertices of degree less than four the rim of the grid. We refer to the vertex set $\{(x, m-1) \mid 0 \leq x<n\}$ as the upper rim and analogously we use lower, left and right rim. For a given vertex $v \in V$ we define $\partial_{x} v:=v_{x}$ and $\partial_{y} v:=v_{y}$, $\bar{\partial}_{x} v:=n-1-v_{x}$, and $\bar{\partial}_{y} v:=m-1-v_{y}$. We also use $\partial v:=\partial_{x} v+\partial_{y} v$ and $\bar{\partial} v:=\bar{\partial}_{x} v+\bar{\partial}_{y} v$.

Parametrised Complexity. A pair $Q=(P, \kappa)$ with $P \subseteq \Sigma^{*}$ and $\kappa: \Sigma^{*} \rightarrow \mathbb{N}$ is called a parametrised problem. A parametrised problem $Q=(P, \kappa)$ admits a problem kernel (or is kernelisable) if there is a polynomial-time algorithm transforming any instance $\mathcal{I}$ of $Q$ into an instance $\mathcal{I}^{\prime}$ such that (i) $\mathcal{I} \in Q \Leftrightarrow$ $\mathcal{I}^{\prime} \in Q$, and (ii) the size of $\mathcal{I}^{\prime}$ (the kernel) is bounded by a computable function $f(\kappa(\mathcal{I}))$. If $f$ is a polynomial, then the problem is said to admit a polynomial (problem) kernel. A parametrised problem is fixed-parameter tractable (or in FPT) if each instance $(x, \kappa)$ can be decided in $f(\kappa(x)) \cdot|x|^{O(1)}$ time, where $f$ is a computable function. A (decidable) parametrised problem is in FPT if and only if it is kernelisable. A parametrised problem that is W-hard is presumably not in FPT.

Further Notation. Let $\mathcal{I}=(G, s, t, p, k)$ be an instance of MSE. We say $\mathfrak{P}$ is a solution for $\mathcal{I}$ if (i) $\mathfrak{P}$ is a multiset of $p s$-t-paths $\left\{P_{1}, \ldots, P_{p}\right\}$, and (ii) $\left|\left\{e \in E \mid \exists 1 \leq i<j \leq p: e \in P_{i} \cap P_{j}\right\}\right| \leq k$. We say that $\mathfrak{P}$ is a trivial solution if $P_{i}=P_{j}$ for all $i, j \in[p]$. An edge is called shared if it occurs in at least two paths of $\mathfrak{P}$.

\section{On Bounded and Holey Grids}

The class of grid graphs appeared frequently in the literature: There is work on grid graphs and related graphs with respect to finding paths [11, 12], routing [4], or structural properties $[1,10]$. In this section we study the complexity of MSE on bounded grids and their subgraphs. We show that MSE is solvable in linear time on bounded grids when both dimensions are either small or large compared to the number $p$ of paths (Section 3.1) and becomes NP-hard for subgraphs of bounded grids (Sections 3.2 and 3.3). We remark that MSE is solvable in lineartime on the class of unbounded grids [6].

\subsection{Bounded Grids}

We fix some instance $\mathcal{I}:=\left(G=G_{n \times m}, s, t, p, k\right)$ for the remainder of the section. Since the problem is invariant under symmetry and swapping $s$ and $t$, we may 
assume $s$ lies left and below of $t$ and $\partial_{x} s \leq \partial_{y} s$. To show optimality of the constructions we regard edge cuts of size less than $p$. Assume $\mathcal{I}$ has a solution $\mathfrak{P}$. We know [6] that after contraction of the shared edges, the graph must allow an $s$ - $t$-flow of value at least $p$. Therefore, every cut smaller than $p$ has to be eliminated by a contraction, that is, it must contain a shared edge.

We distinguish the following different cases depending on the dimensions of the grid in relation to the number $p$ of paths: $p$-small grid $(p>\max \{n, m\})$, $p$-large grid $(p \leq \min \{n, m\})$, and $p$-narrow grids (neither $p$-small nor $p$-large). We leave open whether MSE is solvable in polynomial-time on $p$-narrow grids. However, ongoing work indicates that the question can be answered positively.

On $p$-small grids. If $p>\max \{n, m\}$, then every set of horizontal edges with endpoints having the same coordinates in the grid forms an $s$ - $t$-cut of size smaller than $p$ (analogously for every set of horizontal edges). Hence, intuitively, any set of $p s$-t-paths share an edge for each horizontal or vertical level they cross. Indeed, we prove that every instance on $p$-small grids is a yes instance if and only if it admits the trivial solution.

Lemma 1. If $m<p$ and $n<p$, then we have a solution if and only if $\operatorname{dist}_{G}(s, t) \leq k$.

Proof. Each row and each column between $s$ and $t$ is a cut of size smaller than $p$. Formally, these are rows $\operatorname{row}_{x}:=\{\{(x, y),(x+1, y)\} \mid 0 \leq y<m\}$ for $s_{x} \leq x<t_{x}$ and columns $\operatorname{col}_{y}:=\{\{(x, y),(x, y+1)\} \mid 0 \leq x<n\}$ for $s_{y} \leq y<t_{y}$. All these cuts are disjoint, since neither two rows nor two columns nor a row and a column ever intersect with respect to edges. Their number is $\left(t_{x}-s_{x}\right)+\left(t_{y}-s_{y}\right)=\operatorname{dist}_{G}(s, t)$. So if we have a solution, then $k$ has to be at least $\operatorname{dist}_{G}(s, t)$. Conversely, if $\operatorname{dist}_{G}(s, t) \leq k$, we have a trivial solution by definition.

On $p$-large grids. Compared to the situation on $p$-small grids, $p$-large grids allow for non-trivial solutions. Nevertheless, we prove that the existence of such non-trivial solutions is expressed by arithmetic conditions which can be checked in linear time. These arithmetic conditions basically relate $p, k$, and the positions of $s$ and $t$ relative to the rim of the grid. If $s$ lies sufficiently far away from the corner formed by the left and lower rim, then only every second path in our construction introduces a new shared edge at this part. However, if $s$ lies close to the corner (or if $p$ is large enough), there is a critical number of paths after which every additional path introduces at least one new shared edge. The same happens at the side of $t$. Thus we obtain the following cases.

Lemma 2. Let $p \leq m$ and $p \leq n$. Then there is a non-trivial solution if and only if either

- $p \leq 2(\partial s+2)-\operatorname{deg}(s)$ and $k \geq\left\lceil\frac{1}{2}(p-\operatorname{deg}(s))\right\rceil+\left\lceil\frac{1}{2}(p-\operatorname{deg}(t))\right\rceil$, or

- $2(\partial s+2)-\operatorname{deg}(s)<p \leq 2(\bar{\partial} t+2)-\operatorname{deg}(t)$

and $k \geq p-(\partial s+2)+\left\lceil\frac{1}{2}(p-\operatorname{deg}(t))\right]$, or

- $p>2(\bar{\partial} t+2)-\operatorname{deg}(t)$ and $k \geq 2 p-(\partial s+\bar{\partial} t+4)$.

To simplify the notation for our construction, we introduce the following arrow notation. For $(x, y) \in V$ we define $(x, y) \rightarrow(x+\ell, y):=\{\{(x+i, y),(x+$ $i+1, y)\} \in E \mid 0 \leq i<\ell\}$. Analogously we define $\uparrow, \downarrow$ and $\leftarrow$. We also use the 


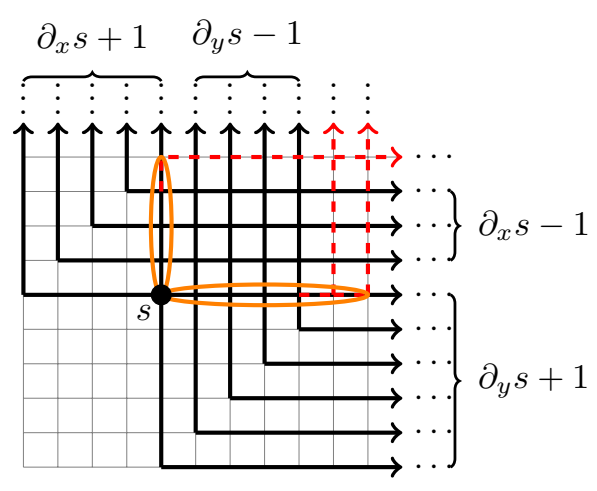

(a) Path construction at vertex $s$.

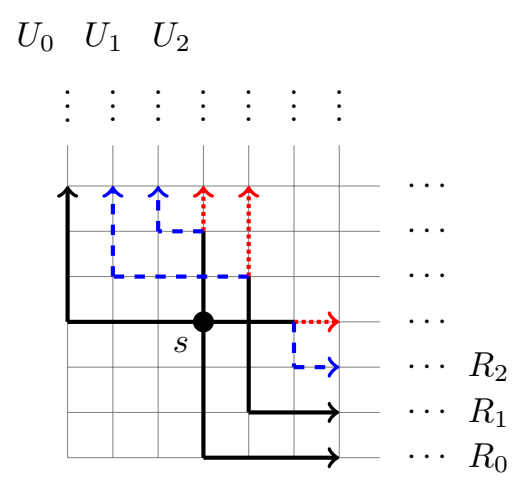

(b) Filling gaps by rerouting.

Figure 1: Sketched aspects of the construction described in the proof of Lemma 2. (a) Path construction at vertex $s$; dashed: one shared edge; black: only a shared edge every two paths; orange ellipses enclose shared edges. (b) Filling gaps by rerouting in the construction; dotted: paths before reconstruction, dashed: rerouted paths for filling the gaps.

concatenation of these expressions such that e.g. $u \rightarrow v \uparrow w:=(u \rightarrow v) \cup(v \uparrow w)$.

Proof. From $s$ we construct path fragments (cf. Fig. 1) going upwards:

$$
\begin{array}{rlrl}
U_{i} & :=s \uparrow\left(s_{x}, s_{y}+i\right) \leftarrow\left(i, s_{y}+i\right) \uparrow(i, m-1-i), & & \leq i<\partial_{x} s, \\
U_{i} & :=s \rightarrow\left(i, s_{y}\right) \uparrow(i, m-1-i), & \partial_{x} s \leq i .
\end{array}
$$

Next, we construct path fragments going to the right:

$$
\begin{array}{rlrl}
R_{i} & :=s \rightarrow\left(s_{x}+i, s_{y}\right) \downarrow\left(s_{x}+i, i\right) \rightarrow(n-1-i, i), & & 0 \leq i<\partial_{y} s, \\
R_{i}:=s \uparrow\left(s_{x}, i\right) \rightarrow(n-1-i, i), & \partial_{y} s \leq i .
\end{array}
$$

To obtain the solution we add the path fragments in the following order. This process is illustrated in Fig. 1a.

A) We start with $U_{0}, U_{\partial_{x} s}, R_{0}$, and $R_{\partial_{y} s}$ which have no shared edge. This yields $\operatorname{deg}(s)$ paths, since some of these are identical if $s$ lies on the rim. Then for $i=1, \ldots, \partial_{y} s-1$ we add $R_{i}$ and $U_{\partial_{x} s+i}$, where $R_{i}$ new adds a shared edge (the other common edges were already shared before). Afterwards we continue adding $U_{i}$ and $R_{\partial_{y} s+i}$ for $i=1, \ldots, \partial_{x} s-1$, where $U_{i}$ adds a shared edge. Thus every other path fragment adds a shared edge. We stop as soon as we have constructed $p$ paths.

B) Continue adding $R_{i}$ for $i=\partial s+1, \ldots, \partial t$ and $U_{j}$ for $j=\partial s+1, \ldots, p-\partial t$ until we reach $p$ paths. Here each single fragment adds another shared edge. For $p \leq 2 \partial s$ we add the following modifications. If $p \leq 2 \partial_{y} s+2$, that is, $R_{\partial_{y} s}$ is the last right-going fragment, extend this fragment downwards such that the endpoints of the $R_{i}$ form a consecutive line. If the $U_{i}$ leave a gap, that is, $U_{j}$ is not part of the construction for some $0<j<\partial_{x} s$, we take the rightmost upgoing fragment and route it leftwards along the first free row, and then continue 
as $U_{j}$. In the end, if necessary, we extend $U_{\partial_{x} s}$ leftwards, like we did with $R_{\partial_{y} s}$. Thus the endpoints of the up-going fragments form a consecutive line as well. These steps do not introduce further shared edges. See Fig. 1b for an illustration.

So in the end we may assume we have constructed fragments $U_{0}, \ldots, U_{u-1}$ and $R_{0}, \ldots, R_{r-1}$ for some $u, r \in \mathbb{N}$ with $u+r=p$. At $t$ we proceed analogously, simply mirrored. Therefore we have $r$ down-going fragments $D_{i}$ and $u$ left-going fragments $L_{i}$. Then we obtain the solution

$$
\mathfrak{P}:=\left\{U_{i} \cup L_{i} \mid i=0, \ldots, u\right\} \cup\left\{R_{i} \cup D_{i} \mid i=0, \ldots, r\right\} .
$$

Feasibility. Furthermore the $R_{i}$ only use the lower $r$ rows of the grid whereas the $L_{i}$ use the upper $u$ rows. Since $r+u=p \leq m$, these do not intersect, that is, we do not get further shared edges. The same holds for the $U_{i}$ and $D_{i}$, since $p \leq n$.

Let $k_{s}$ and $k_{t}$ denote the number of shared edges used to construct the path fragments at $s$, and at $t$ respectively. Thus, we have a solution if $k \geq k_{s}+k_{t}$.

If $p \leq 2(\partial s+2)-\operatorname{deg}(s)$, then we only use part A. From the $\operatorname{deg}(s)$-th path to the $p$-th path, every other path adds a new shared edge, so $k_{s}=\left\lceil\frac{1}{2}(p-\operatorname{deg}(s))\right\rceil$. Furthermore, $\partial s \leq \bar{\partial} t$, so at $t$ we also only use part A. This implies $k_{t}=$ $\left\lceil\frac{1}{2}(p-\operatorname{deg}(t))\right\rceil$. Hence $k \geq k_{s}+k_{t}=\left\lceil\frac{1}{2}(p-\operatorname{deg}(s))\right\rceil+\left\lceil\frac{1}{2}(p-\operatorname{deg}(t))\right\rceil$.

If $2(\partial s+2)-\operatorname{deg}(s)<p \leq 2(\bar{\partial} t+2)-\operatorname{deg}(t)$, we still only use part A at $t$ getting $k_{t}=\left\lceil\frac{1}{2}(p-\operatorname{deg}(t))\right\rceil$. But at $s$ we also use part B. Assume that $s$ lies in the interior of the grid. Then, when completely executing part A, we use $\partial s-2$ shared edges to construct $2 \partial s$ paths. This leaves $k_{s}-(\partial s-2)$ shared edges for part B. Each of those allows for another path. So we obtain the condition $p=\partial s+2+k_{s}$. If $s$ lies on the rim or in the corner, then the argument differs slightly, but the condition is the same. So overall we get the condition $k \geq k_{s}+k_{t}=p-(\partial s+2)+\left\lceil\frac{1}{2}(p-\operatorname{deg}(t))\right\rceil$.

Finally, if $p>2(\bar{\partial} t+2)-\operatorname{deg}(t)$, then we use part B at both $s$ and $t$. Thus we have $p=\partial s+2+k_{s}=\bar{\partial} t+2+k_{t}$. By adding these equalities we obtain $k_{s}+k_{t}=2 p-(\partial s+\bar{\partial} t+4) \leq k$. So the solution is feasible.

Optimality. We only give a lower bound for the number $k_{s}$ of shared edges at $s$. The bound for $k_{t}$ follows analogously, which then gives the desired bound for $k$.

During part A) of the construction, each contraction may increase the degree of $s$ by at most 2 . Hence $p \leq \operatorname{deg}(s)+2 k_{s}$, which shows $k_{s} \geq\left\lceil\frac{1}{2}(p-\operatorname{deg}(s))\right\rceil$.

For $p \geq 2(\partial s+2)$ we present a number of cuts of size $p-1$. We use rectangles containing $s$, whose right upper corners move along a diagonal. Formally, these are cut $_{i}:=\{\{(i, y),(i+1, y)\} \mid y \leq p-3-i\} \cup\{\{(x, p-3-i),(x, p-2-i)\} \mid x \leq i\}$ for $i=s_{x}, \ldots, p-3-s_{y}$. Assume that $t$ lies inside one of those rectangles. Then cut $_{i}$ for $s_{x} \leq i<t_{x}$ and $p-3-t_{y}<i \leq p-3-s_{y}$ are $s$ - $t$-cuts of size $p-1$, and these are $\operatorname{dist}_{G}(s, t)$ many. In this case we need $k \geq \operatorname{dist}_{G}(s, t)$ which only allows the trivial solution. So we may assume that $t$ lies outside all of these rectangles. Thus there are $p-2-\partial s$ many of these cuts and they separate $s$ and $t$. Furthermore they are pairwise disjoint. So we get $k_{s} \geq p-2-\partial s$.

Altogether, our construction is optimal. 


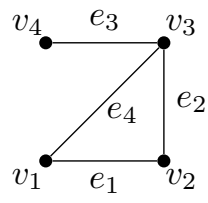

(a) Example graph.

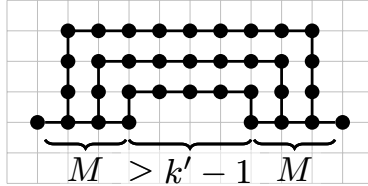

(b) Rainbow gadget.

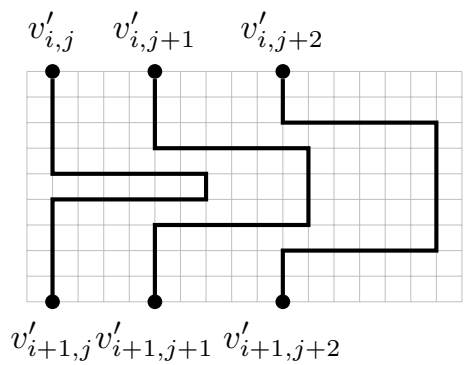

(c) Snake-chains.

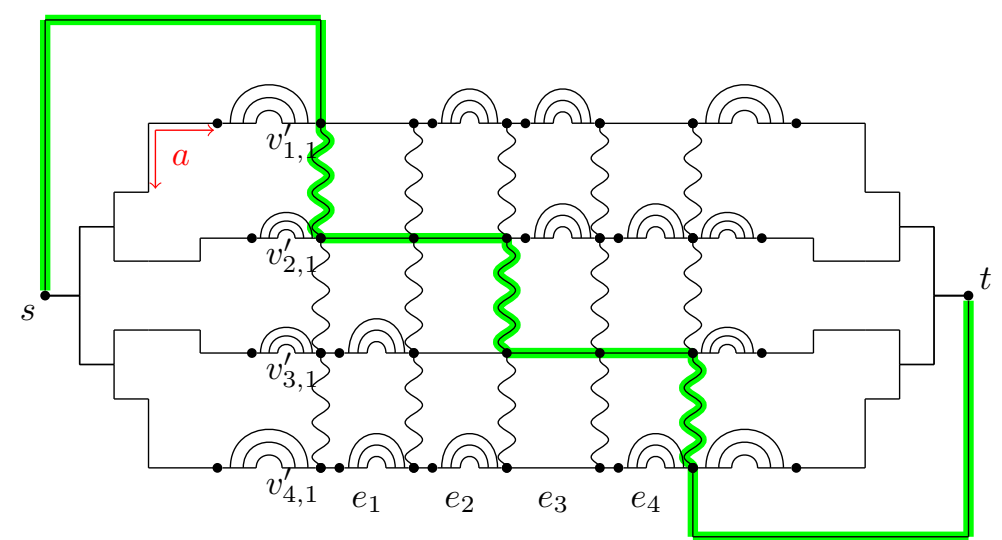

(d) Sketch of the graph obtained in the proof of Theorem 1.

Figure 2: An exemplified illustration of the construction in the proof of Theorem 1. (a) A graph representing an example instance of VerTEx Cover. (b) An illustration of (the grid-embedding of) the rainbow gadget. (c) An illustration of snake-chains. (d) Sketch of the holey grid constructed in the proof of Theorem 1, exemplified with the instance represented by (a). The highlighted path indicates the validation path.

\subsection{Holey Grids}

In the previous section we proved that MSE is solvable in linear time on small and large (compared to the number $p$ of paths) bounded grids. In this section we study the complexity of MSE on subgraphs of bounded grids, which we call holey grids and show that the problem is NP-hard on this graph class. To this end we reduce from the well known VERTEX COVER problem which is, given a graph $G$ and a natural number $k \in \mathbb{N}$, to decide whether there exists $U \subseteq V$ with $|U| \leq k$ such that $\forall e \in E: e \cap U \neq \emptyset$. More precisely, we use that VERTEX COVER remains NP-complete on graphs with maximum degree three [9]. Note that our reduction adapts the idea of a reduction used in previous work [8].

Theorem 1. MSE on holey grids is NP-hard.

Proof. Given an instance $\mathcal{I}_{\mathrm{Vc}}:=(G=(V, E), k)$ of VerTex Cover with $\Delta(G) \leq$ 3 , we compute an equivalent instance $\mathcal{I}_{\mathrm{MSE}}:=\left(G^{\prime}=\left(V^{\prime}, E^{\prime}\right), s, t, p, k^{\prime}\right)$ of MSE on holey grids in polynomial time. We assume that $|V|$ is a power of two (oth- 
erwise we add isolated vertices until it is).

Figure $2 d$ illustrates the graph obtained by applying the following transformation to the graph shown in Figure 2a. The main part of the construction is a structure we refer to as meta-grid. The meta-grid encodes the vertex-edge incidence matrix of the original graph. We assume that the obtained graph to be embedded as shown in Figure 2d, which serves as a reference when we use the terms "left", "right", "up", and "down". For construction purposes, we refer to paths with $\ell+1$ vertices as $\ell$-chains or chains of length $\ell$. Whenever a chain is added in the construction, all vertices except the two end-vertices are new.

The main component in the construction is a gadget called rainbow (cf. [8]), see Figure 2b. Figure 2b also shows that this gadget is a subgraph of a bounded grid. We use rainbow gadgets where the number of vertices in each band in the spectrum of the rainbow is larger than the number of allowed shared edges. This allows the rainbow gadget to restrict the number of paths that can be routed through it to at most the number of bands in the spectrum. Note that in any rainbow that is satiated with $M$ paths $2 M-2$ edges are shared. We call the number of shared edges in a rainbow the rainbow-offset.

We define $M$ and a few other values we need in order to build the graph $G^{\prime}$ in the following:

$$
\begin{aligned}
M & :=2(|E|+1)+2 ; \quad \text { trees }:=2 \cdot\left(|V| \cdot \log _{2}(|V|)-2+2 k\right) ; \\
c & :=10 ; \quad c^{\prime}:=2|V|+|E| \cdot|V|-2|E| ; \quad b:=2 \cdot M \cdot c^{\prime}+1 ; \\
a_{0} & :=\frac{|V|-1}{2}(M+c-2)-\log |V| ; \quad a:=\max \left(a_{0},|E|^{3}, b^{2}\right) .
\end{aligned}
$$

Here, $c^{\prime}$ is the number of rainbow gadgets we construct. The values $a, a_{0}$, and $b$ are chosen to ensure certain constraints when routing paths and sharing edges while $c$ can be understood as a scaling constant used to avoid intersections. Why the values are chosen in this way will become clear later in the proof. Next we set $p:=k \cdot M+(|V|-k)+1$ for the number of paths and $k^{\prime}:=$ $k \cdot(2 a+b|E|)+$ trees $+c^{\prime}(2 M-2)$ for the number of shared edges in $\mathcal{I}_{\mathrm{MSE}}$.

In the following we describe the construction of the meta-grid. First, we create a grid of vertices, without any edges, that has $|V|$ rows and $|E|+1$ columns. We fix an ordering $v_{1}, \ldots, v_{|V|}$ on the set $V$ of vertices and use it to identify each row of the grid with a vertex from $G$. Analogously, we fix an order $e_{1}, \ldots, e_{|E|}$ on the edge set $E$ and use it to identify each space between two consecutive columns of the grid with an edge in $G$. From here on we will refer to these spaces as columns.

The first vertex in row $i$ is denoted $v_{i, 1}^{\prime} \in V^{\prime}$, refer to Figure 2d, the second one is denoted $v_{i, 2}^{\prime}$, and so on. If vertex $v_{i}$ is incident to edge $e_{j}$ in $G$, then vertices $v_{i, j}^{\prime}$ and $v_{i, j+1}^{\prime}$ are connected by a chain of length $b$. If $v_{i}$ is not incident to $e_{j}$ in $G$, then vertices $v_{i, j}^{\prime}$ and $v_{i, j+1}^{\prime}$ are connected by a chain of length $b$ followed by a rainbow. This completes the construction of the rows.

We embed the structure we just created in a grid such that the first vertices are vertically aligned and have vertical distance of $M+c$. Now we connect each vertex $v_{i, j}^{\prime}$ with $i<|V|$ and $j<|E|$ with its respective lower neighbour, that is, vertex $v_{i+1, j}^{\prime}$, by so-called snake-chains of length at least $k^{\prime}+1$ (the wavy vertical lines in Figure 2d). Note that these vertices do not necessarily lie above each other. The snake-chains are constructed as follows (refer to Figure 2c for 
an illustration).

In every row except the lowest one, we start with the left most snake-chain. We first route it four steps down, then $k^{\prime}$ steps to the right, one down, left again until we are above its end-vertex which we then join it to by a vertical path. Then every further snake-chain is routed the following way: down by the maximum possible number of steps (at most four) such that no previous snake-chain is intersected, then $k^{\prime}$ to the right, then the minimum necessary number of steps down, such that the snake-chain can be extended to the left without intersecting a previous snake-chain until it can be routed downwards until it meets its end-vertex.

Note that the above description implies that we reduce the number of steps that a snake-chains is routed downwards every time the previous column did not contain a rainbow. After a rainbow is encountered we start with four steps down again. Since $G$ has a maximum degree of three, there are at most three columns in every row without a rainbow, so after at most four consecutive snake-chains we encounter a rainbow in the next column. This way the snake-chains do not intersect or touch each other and the constant $c>2 \cdot 4+1$ ensures that they also do not intersect any rainbows from the next row.

Now we add a source vertex $s$ to the left of the meta-grid and construct a complete, binary tree of height $\log _{2}|V|$ with $s$ as its root and with $|V|$ leaves pointing in direction of the grid. We construct this tree in such a way that all vertices of the same level lie in the same column of the grid and from one leaf to the next we have distance two in the grid. This is possible since the number of vertices in $G$ is a power of two. To make this tree embeddable into a grid we replace every edge by a chain of the minimal required length running along the grid structure. We connect the uppermost leaf to the first row of the meta-grid in a way such that the vertical distance between this leaf and $v_{1,1}^{\prime}$ is exactly $a_{0}$. More specifically, we add a chain up and to the right until it has length $a$, then add a rainbow of sufficient length and connect it to $v_{1,1}^{\prime}$. Each leaf of the tree is connected by a chain of length $a$ and a following rainbow to one of the vertices in the first column of the meta-grid such that the order of the leaves and the vertices is the same. The length $a$ is chosen such that all the chains have the same length. To avoid intersections in the $a$-chains these go right first: the chain leading to the row corresponding to $v_{i}$ is routed $i-1$ steps to the right if $i<\frac{|V|}{2}$ and $|V|-i$ otherwise. Then the chains go up/down to their row and then right until they have length $a$. Note that this tree is symmetrical in the end since we work on an even number of vertices.

The same is done on the right side: we add a vertex $t$ and a binary tree to its left with $t$ being the root and the leaves are connected to the vertices in the last column of the meta-grid by a chain of length $a$ and a rainbow. If the construction of the snake-paths causes some of the snake-paths to "stick out" to the right, then we extend the paths in the rainbows at the leaves of $t$ as far as necessary to ensure that nothing intersects. The length of these rainbows is also used to align the leaves of the tree on this side.

Finally, we add chains of length at least $k^{\prime}+1$, the outer-grid chains, one connecting $s$ to $v_{1,1}^{\prime}$ and the other connecting $v_{|V|,|E|+1}^{\prime}$ to $t$.

Intuitively, the correctness is shown as follows. Recall that $p:=k \cdot M+$ $(|V|-k)+1$. We know that we can route at most $M$ paths through a rainbow, this we have to do $k$ times. So we can pick $k$ of the $|V|$ rows and route $M$ paths 
through each. We route a single path through each of the remaining $|V|-k$ rows. Now we have to route one additional path, which has to use the outergrid chains and the snake-chains. This path will verify that the $k$ rows we chose to route $M$ paths through correspond to vertices of $G$ that constitute a vertex cover. Then each column corresponding to an edge of $G$ has at least one row where we have a fully shared chain and no rainbow. So the remaining path can be routed through those chains and use the snake-chains to switch between rows. Of course, $k^{\prime}$ is chosen in a way that we are forced to use the described approach and that there is no solution if $G$ does not have a vertex cover of size $k$. We claim that $G$ has a vertex cover of size $k$ if and only if $G^{\prime}$ has $p$ paths from $s$ to $t$ sharing at most $k^{\prime}$ edges.

$(\Rightarrow)$ Let $\mathrm{VC} \subseteq V$ be a vertex cover of size at most $k$. Without loss of generality we assume $|\mathrm{VC}|=k$. Then for each $v \in \mathrm{VC}$, we route $M$ paths from $s$ via the chain of length $a$ and through the following rainbow leading to the corresponding vertex $v^{\prime}$. This way each $M$ paths cause $a$ shared edges within the corresponding chain plus the rainbow-offset of $2 M-2$ edges, so in total $k \cdot(a+2 M-2)$. For all other vertices we route only one path this way. Doing so we cause every edge in the tree on the side of $s$ to be shared except on its lowest level, where only $k$ branches of the tree are shared, notice that this yields exactly trees $/ 2$ shared edges. So the paths cause $a \cdot k+k(2 M-2)+$ trees $/ 2$ shared edges before reaching the meta-grid. Next we route all these paths horizontally through our meta-grid yielding another $k \cdot b \cdot|E|+k \cdot(2 M-2)$ shared edges.

To route the paths from the meta-grid to $t$ we get additional $k \cdot(a+2 M-$ $2)+$ trees $/ 2$ shared edges. So we have $k \cdot M+(|V|-k)$ paths sharing $k \cdot(2 a+$ $b|E|)+$ trees $+c^{\prime}(2 M-2) \leq k^{\prime}$ edges. The number trees therefore describes exactly the number of edges in the trees at $s$ and $t$ that are shared.

Next we route one additional path $P$ without sharing any additional edges. Starting at $s$ we route $P$ along the outer-grid chain linking $s$ to $v_{1,1}^{\prime}$, the first vertex in the first row. From there $P$ has to pass the columns of the meta-grid. Since VC is a vertex cover there is a covering vertex for every edge. So for every column we take the vertex covering it and route the path to the corresponding row using the snake-chains. Since the vertex is part of the vertex cover the chain crossing this column is already shared, so we can use it to route $P$ on to the next column. After $P$ has crossed the meta-grid in this fashion it can be routed to the lowest row via the snake-chains and then via the outer-grid chain leading to $t$.

So $G^{\prime}$ allows for $k \cdot M+(|V|-k)+1 s$-t-paths sharing $k^{\prime} \leq k \cdot(2 a+b|E|)+$ trees $+c^{\prime}(2 M-2)$ edges. Hence, $\mathcal{I}_{\mathrm{MSE}}$ a yes-instance.

$(\Leftarrow)$ Assume that $G^{\prime}$ has a solution $\mathfrak{P}$, a set of $s$ - $t$-paths with $|\mathfrak{P}|=p$ sharing at most $k^{\prime}$ edges. First consider how the paths leave $s$. There are $|V|+1$ ways to get from $s$ to the meta-grid. One of those is the outer-grid chain which can only contain one path since it has length $k^{\prime}+1$. So $p-1=k \cdot M+(|V|-k)$ paths have to be routed through the tree. Also every rainbow can contain at most $M$ paths. And due to $k^{\prime}<2 a(k+1)$ at most $2 k$ of the $a$-chains in $G^{\prime}$ can be shared, which means $k$ of the $a$-chains connected to $s$ and $k$ of the $a$-chains connected to $t$. Consequently, we get that $k$ of the rainbows connected to the first column of the meta-grid contain $M$ paths each. Let $v_{i_{1}, 1}^{\prime}, \ldots, v_{i_{k}, 1}^{\prime}$ be the first vertices from these rows. The other rainbows connected to the first column then contain exactly one path.

Next we consider what happens inside the meta-grid. Since the snake-chains 
are the only way to leave a row and those cannot be shared, at most $2(|E|+1)$ paths can leave a row. So in the last column at least 2 paths are still routed through the rainbow connected to these rows and then via the $a$-chain to $t$. We can do the same maths as before on the $t$ side to get that these rainbows have to contain $M$ paths as well. Hence, each of this $k$ rows induces at least $2 a+b|E|$ shared edges. Also trees many shared edges are induced within the trees by construction. Note that $b>c^{\prime} \cdot(2 M-2)$. Hence, there are less than $b$ shared edges left.

Now let $\mathrm{VC}:=\left\{v_{i_{1}}, \ldots, v_{i_{k}}\right\}$. Assume towards a contradiction that VC is not a vertex cover. Then there is a column with a rainbow in every of the $k$ rows corresponding to the vertices in VC. That means that the path that is routed via the outer-grid chain connected to $s$ cannot pass this column without sharing additional $b$ edges, but our budget does not suffice for this. This yields a contradiction to $G^{\prime}$ being a yes-instance.

\subsection{Manhattan-like Acyclic Digraphs}

In the previous section, we proved that MSE is NP-hard on holey grids, i.e. subgraphs of a bounded grid. Along the line, in this section we prove that the directed version, DMSE, is NP-hard on the graph class of acyclic directed holey grids (we refer to this class by Manhattan DAGs). We remark that inspired by the street design of Manhattan, New York City, directed bounded grids (referred to as Manhattan street networks) are considered in the literature, also in the context of routing $[13,17]$.

Observe that MSE reduces to DMSE by replacing each edge $\{u, v\}$ by antiparallel arcs $(u, v),(v, u)$. The correctness of the reduction follows immediately from the following.

Lemma 3. Let $(G, s, t, p, k)$ be an instance of DMSE. If $\mathfrak{P}$ is a solution for this instance where two paths $P_{A}$ and $P_{B}$ use $e=(u, v) \in E$ and its inverted arc $e^{\prime}=(v, u) \in E$, then we can find a solution $\mathfrak{P}^{\prime}$ for the same instance that does not use both of these arcs.

Proof. The idea is to split both paths by removing those two edges and connect the beginning of the first path to the trailing part of the second path and vice versa, retaining a solution for the instance.

We first introduce some notation: When $P$ is a directed path and $u, v \in P$ occur in that order, then we denote the subpath of $P$ that starts at $u$ and ends at $v$ by $P[u, v]$. If $P_{1}$ and $P_{2}$ are two paths and the last vertex of $P_{1}$ is the same as the first vertex of $P_{2}$, then we write $P_{1} \cdot P_{2}$ for the path resulting by the union of the two paths and removing all cycles.

Assume $(G, s, t, p, k)$ has a solution $\mathfrak{P}$ with $e \in \mathrm{P}_{A} \in \mathfrak{P}$ and $e^{\prime} \in \mathrm{P}_{B} \in \mathfrak{P}$. Then we can split $P_{A}$ and $P_{B}$ into subpaths as follows: $P_{A}=P_{A}[s, u] \cdot e \cdot P_{A}[v, t]$ and $P_{B}=P_{B}[s, v] \cdot e^{\prime} \cdot P_{B}[u, t]$.

Now, by replacing path $P_{A}$ by $P_{A}^{\prime}:=P_{A}[s, u] \cdot P_{B}[u, t]$ and $P_{B}$ by $P_{B}^{\prime}:=$ $P_{B}[s, v] \cdot P_{A}[v, t]$, we are able to retain a solution in which these two paths do not use $e$ and $e^{\prime}$ any more.

We can repeat this until one of the two edges is not used any more by any of the paths in the solution, yielding the desired $\mathfrak{P}^{\prime}$. 
However, the directed graph obtained in the reduction is not acyclic. We show next that DMSE remains hard even on acyclic directed holey grids. On a high level, we adapt the construction presented in the proof of Theorem 1 . We then direct the edges from left to right, from $s$ towards $t$. Finally, we duplicate the horizontal chains (snake chains) and direct one upwards and one downwards.

Theorem 2. DMSE on Manhattan DAGs is NP-hard.

Proof. We adapt the reduction for holey grids given in the proof of Theorem 1 . To obtain a directed graph, we replace every edge in the corresponding construction by a directed edge: First we ignore the vertical snake-chains and handle the remaining edges. Each horizontal edge is directed to the right, rainbows go up, then right, then down. The tree edges are directed away from $s$ and towards $t$. Because each snake-chain has to offer both vertical directions in the new construction without creating a cycle, they are constructed as follows. We replace the undirected snake-chains of the original construction by two snakechains separated by single edges in their start and end. The single edges are directed to the right, the left of the new snake-chains is directed downwards and the right one upwards. We call a snake-chain outgoing from the row in which it starts and ingoing to the row where it ends. To ensure that we still have enough space for the additional snake-chain, we increase the distance between the rows by defining $c:=20$.

Let $\mathcal{I}_{\mathrm{VC}}:=(G, k)$ be an instance of VerTEX Cover and $\mathcal{I}_{\mathrm{MSE}}^{\prime}:=\left(G^{\prime}, s, t, p, k^{\prime}\right)$ be an instance of MSE constructed using the reduction for holey grids. From this we construct an instance $\mathcal{I}_{\text {DMSE }}^{\prime \prime}:=\left(G^{\prime \prime}, s, t, p, k^{\prime \prime}\right)$ of DMSE according to the additional steps described above. In the construction for holey grids there are chains of length $b$ inside the meta-grid. Due to the additional single edges connecting each pair of snake-chains, we get chains of length $b^{\prime}:=b+1$ in this construction. Because $b$ is used in the definition of $k^{\prime}$, the incrementation of $b$ leads to $k^{\prime \prime}:=k^{\prime}+k \cdot|E|$.

We claim that $G$ has a vertex cover of size $k$ if and only if $G^{\prime \prime}$ has $p$ paths from $s$ to $t$ sharing at most $k^{\prime \prime}$ edges.

$(\Rightarrow)$ This direction of the proof works analogously to the reduction for holey grids using the new instance $\mathcal{I}_{\mathrm{DMSE}}^{\prime \prime}$ instead of $\mathcal{I}_{\mathrm{MSE}}^{\prime}$. The first $k \cdot M+(|V|-k)$ paths are routed in the same way. Then again we route one additional path by always choosing the corresponding outgoing snake-chain. Since we have a vertex cover, this does not share further edges.

$(\Leftarrow)$ Let $\mathfrak{P}$ be a solution for $\mathcal{I}_{\text {DMSE }}^{\prime \prime}$. The difference to the reduction for holey grids lies in the number of snake-chains that is doubled in the new construction. Now, after sharing trees edges (for definition of trees see the proof of Theorem 1 ) in the binary trees at $s$ and $t$, at most $2 k$ of the $a$-chains between the binary trees and the meta-grid in $G^{\prime \prime}$ can be shared.

Since only the outgoing snake-chains can be used to leave a row, we can argue again that there are $k$ rows in the meta-grid containing more than one path, each one inducing at least $2 a+b^{\prime}|E|$ shared edges. Let VC be the set vertices corresponding to these rows. If VC is no vertex cover, then we need to share another $b^{\prime}$-chain in the construction adding additional $b^{\prime}$ shared edges, which is a contradiction to $\mathcal{I}_{\text {DMSE }}^{\prime \prime}$ being a yes-instance. 


\section{The Nonexistence of Polynomial Kernels}

In this section, we consider MSE from a parametrised complexity point of view. MSE is kernelisable but does not admit a polynomial problem kernel when it is parametrised by the number $p$ of paths, unless NP $\subseteq$ coNP/poly [7]. We strengthen the latter result and complement the intractability of MSE on planar graphs by showing the following.

Theorem 3. Unless NP $\subseteq$ coNP/poly, MSE with parameter $\kappa(G, s, t, p, k):=$ $p+k+\Delta(G)+\operatorname{diam}_{G}+\operatorname{tw}(G)$ does not admit a polynomial kernel, even on planar graphs.

In order to prove Theorem 3, we use a so-called OR-cross-composition due to Bodlaender et al. [5]. Therein, one uses a polynomial equivalence relation $\mathcal{R}$ which is an equivalence relation that is decidable in polynomial time and for each finite set $S$, the number of equivalence classes with respect to $\mathcal{R}$, that is, $\left|\left\{[s]_{\mathcal{R}} \mid s \in S\right\}\right|$, is polynomially bounded in the size of the largest element in $S$.

Definition 1 (OR-cross-composition [5]). Let $L \subseteq \Sigma^{*}$ be some problem and $Q=(P, \kappa)$ with $P \subseteq \Sigma^{*}$ and $\kappa: \Sigma^{*} \rightarrow \mathbb{N}$ be some parametrised problem. Furthermore, let $\mathcal{R}$ be a polynomial equivalence relation on $\Sigma^{*}$. An OR-crosscomposition is an algorithm that gets instances $\mathcal{I}_{1}, \ldots, \mathcal{I}_{q}$ of $L$ as input, all of them belonging to the same equivalence class of $\mathcal{R}$, and outputs an instance $\mathcal{I}$ of $Q$ such that

- $\mathcal{I} \in P$ if and only if there is at least one $i$ such that $\mathcal{I}_{i} \in L$ and

- $\kappa(\mathcal{I})$ is polynomially bounded in $\max \left\{\left|\mathcal{I}_{i}\right| \mid i=1, \ldots, q\right\}+\log q$.

If there is an OR-cross-composition from an NP-hard problem $L$ to some parametrised problem $Q$, then $Q$ does not admit a polynomial-size kernel, unless NP $\subseteq$ coNP/poly [5]. Using this result, we give an OR-cross-composition to prove Theorem 3. Our construction contains binary trees and we use the following structural result on binary trees with respect to MSE.

Lemma 4. Let $T$ be a balanced, binary and complete tree of height $h$ with root $s$, where additionally all leaves are identified with the target $t$. Then the only solutions for an MSE-instance $(T, s, t, p, k)$ with $p \geq h+3$ and $k \leq h$ are to share a complete path from $s$ to some leaf, which is only possible for $k=h$.

Proof. The idea is again that the contraction of the shared edges must allow a flow of value $p$ from $s$ to $t$ in any solution.

Initially $s$ has degree two. Each contraction of an edge may only increase the degree by one. So after $k$ contractions the degree is at most $h+2<p$, except if the contraction identifies $s$ with $t$. This can only happen if a complete path from $s$ to $t$ is shared. Since such a path has length $h$ this is only possible for $k \geq h$. Due to the condition $k \leq h$ there are no other shared edges and $k=h$.

Next we prove the main result of this section.

Proof of Theorem 3. We apply the OR-cross-composition framework with MSE on planar graphs where $s$ and $t$ lie on the outer face as input problem. The NP-hardness of this problem is shown in Theorem 1 since in the reduction $s$ and $t$ are on the outer face. 


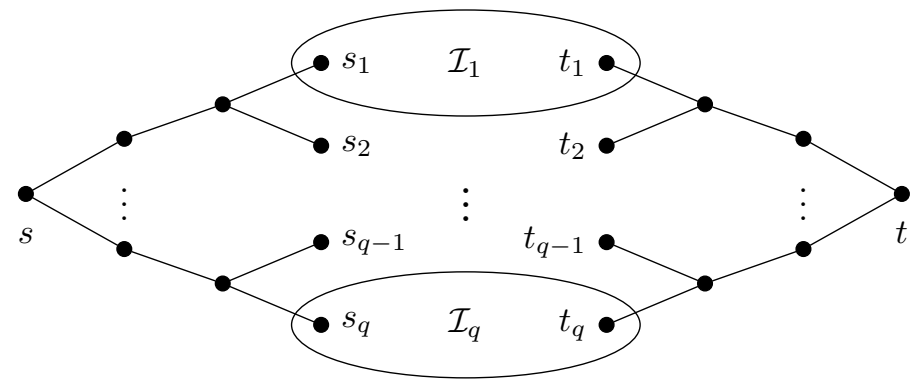

Figure 3: Construction of $\mathcal{I}$ via an OR-cross-composition. The instances are connected by complete binary trees with roots $s$ and $t$, respectively.

We say an instance $(G, s, t, p, k)$ of MSE is malformed if $\operatorname{dist}_{G}(s, t) \leq k$ (trivial yes-instances), if $s$ and $t$ are not connected, if $p \geq 2 \cdot|E(G)|$ and $k<$ $\operatorname{dist}_{G}(s, t)$ (trivial no-instances), or if $p \leq 2$. Note that in the last case we can decide the instance in polynomial time, since the problem is fixed-parameter tractable with respect to $p$ [7]. Hence we can decide each malformed instance in polynomial time.

We define the equivalence relation $\mathcal{R}$ as follows: two instances $(G, s, t, p, k)$ and $\left(G^{\prime}, s^{\prime}, t^{\prime}, p^{\prime}, k^{\prime}\right)$ are $\mathcal{R}$-equivalent if both are malformed or if $p=p^{\prime}$ and $k=$ $k^{\prime}$. Observe that $\mathcal{R}$ is a polynomial equivalence relation.

Let $\mathcal{I}_{i}=\left(G_{i}, s_{i}, t_{i}, p, k\right)_{1 \leq i \leq q}$ be non-malformed $\mathcal{R}$-equivalent instances of MSE. We assume $q$ to be a power of 2 (as otherwise we duplicate instances until it is). We first construct a complete binary tree $T_{s}$ rooted in $s$ with depth $\log (q)$ such that the $s_{i}$ are the leaves of $T_{s}$, occurring in their canonical order. Conversely, we construct a tree $T_{t}$ with root $t$ and leaves $t_{i}$. We subdivide each edge in $T_{s}$ and $T_{t}$ to obtain paths of length $k+1$. In this way we obtain a new graph $G=(V, E)$ with $V:=V\left(T_{s}\right) \cup V\left(T_{t}\right) \cup \bigcup_{i=1}^{q} V\left(G_{i}\right)$ and $E:=E\left(T_{s}\right) \cup$ $E\left(T_{t}\right) \cup \bigcup_{i=1}^{q} E\left(G_{i}\right)$. Furthermore, we define the new parameters $p^{\prime}:=p+\log (q)$ and $k^{\prime}:=2 \log (q) \cdot(k+1)+k$ and get the instance $\mathcal{I}:=\left(G, s, t, p^{\prime}, k^{\prime}\right)$, see Fig. 3 . Now we claim that $\mathcal{I}$ is a yes-instance if and only if there is an $\mathcal{I}_{y}$ with $1 \leq y \leq q$ that is a yes-instance.

In the trees let $P_{x}$ denote the path from $s$ to $s_{x}$ in $T_{s}$ and let $Q_{x}$ denote the path from $t_{x}$ to $t$ in $T_{t}$ for every $1 \leq x \leq q$.

$(\Leftarrow)$ Assume that $\mathcal{I}_{y}$ is a yes-instance with solution $\mathfrak{P}_{y}$. We route $p$ paths along $P_{y}$, through $\mathcal{I}_{y}$ and along $Q_{y}$ sharing at most $k$ edges within $\mathcal{I}_{y}$ and additional $2 \log (q) \cdot(k+1)$ edges in the trees. Thus in $\mathcal{I}$ at most $k^{\prime}$ edges are shared. Note that every other instance $\mathcal{I}_{i}$ allows for at least one path, since $s$ and $t$ are connected. So from each vertex of $P_{y}$, except $s_{y}$, we route an additional path through one of the remaining instances. Thus we get $\log (q)$ additional paths not sharing any additional edge. So we have $p^{\prime}$ paths and therefore a solution for $\mathcal{I}$.

$(\Rightarrow)$ Assume the constructed instance $\mathcal{I}$ has a solution $\mathfrak{P}$. Note that in $T_{s}$ and $T_{t}$ we share at most $2 \log (q)$ of the $(k+1)$-paths, but we have to route $p^{\prime} \geq \log (q)+3$ paths, since $p \geq 3$. By Lemma 4 this implies that there exist $1 \leq y, x \leq q$ such that $P_{y}$ and $Q_{x}$ are completely shared. At each vertex of $P_{y}$ only one path from $\mathfrak{P}$ may branch off, which implies that only $\log (q)$ paths do so. Hence, at least $p$ paths are routed through $P_{y}$, leaving the instance at 
$t_{y}$. So $Q_{y}$ must be shared, which implies $y=x$. Note that $\mathfrak{P}$ therefore shares $2 \log (q)(k+1)$ outside of $\mathcal{I}_{y}$. That leaves $k=k^{\prime}-2 \log (q)(k+1)$ edges that can be shared inside of $\mathcal{I}_{y}$. So $\mathfrak{P}$ restricted to $\mathcal{I}_{y}$ is a solution for $\mathcal{I}_{y}$.

This concludes the proof that $\mathcal{I}$ has a solution if and only if there is an $1 \leq i \leq q$ such that $\mathcal{I}_{i}$ has a solution. Finally, we observe that $p^{\prime}$ and $k^{\prime}$ are polynomially bounded in $p+\log (q)$ and $k+\log (q)$. Since we only added binary trees, the maximum degree $\Delta$ is increased by at most two. For all $1 \leq x, y \leq q$ and every two vertices in the instances $\mathcal{I}_{x}$ and $\mathcal{I}_{y}$ there is a connecting path via $s_{x^{-}-s^{-} s_{y}}$ of length at most $\operatorname{diam}_{G_{x}}+2 \log (q)+\operatorname{diam}_{G_{y}}$. For all vertices in $T_{s}$ and $T_{t}$ we have a connecting path by going to $s$, then through $\mathcal{I}_{1}$ and finally via $t$ to the desired vertex. This path has length at most $4 \log (q)+\operatorname{diam}_{G_{1}}$. Hence, the diameter of $G$ is at most $4 \log (q)+\max \left\{\operatorname{diam}_{G_{i}} \mid i=1, \ldots, q\right\}$, which is polynomial in the input size and $\log (q)$. The treewidth of $G$ is upper-bounded by $3 \cdot \operatorname{diam}_{G}$, because the graph is planar [16]. (Alternatively, there also is a tree decomposition of $G$ of treewidth at most $2 \log (q)+\max \left\{\operatorname{tw}\left(G_{i}\right): i=1, \ldots, q\right\}$.)

So $\kappa(\mathcal{I})$ is polynomially bounded by $\max \left\{\kappa\left(\mathcal{I}_{i}\right): i=1, \ldots, q\right\}+\log (q)$. It follows that MSE parametrised by $k+p+\Delta+$ diam + tw does not admit a polynomial kernel, even on planar graphs.

Recall that DMSE is NP-hard on planar acyclic digraphs with $s$ and $t$ lying on the outerface (Theorem 2). Hence, replacing the input instances by instances from DMSE on the aforementioned graphs, and directing the remaining edges in the trees away from $s$ and towards $t$ allows us to also exclude polynomial kernels for DMSE parametrised ${ }^{1}$ by $p+k+\Delta_{\text {in }}(G)+\Delta_{\text {out }}(G)$.

Corollary 1. Unless NP $\subseteq$ coNP/poly, DMSE on planar acyclic digraphs with parameter $\kappa(G, s, t, p, k):=p+k+\Delta_{\text {in }}(G)+\Delta_{\text {out }}(G)$ does not admit a polynomial kernel.

\section{Conclusion}

On the positive side, we proved that Minimum Shared Edges on bounded grids is solvable in linear time when both dimensions are either small or large compared to the number $p$ of paths. On the negative side, we proved that MSE becomes NP-hard on subgraphs of the bounded grid, even if the subgraph is directed and acyclic, and that it does not allow for polynomial kernels on planar graphs when parametrised by a combined parameter $k+p+\Delta+$ diam + tw, unless $\mathrm{NP} \subseteq$ coNP/poly.

We conjecture that MSE on $p$-narrow grids is solvable in polynomial time. In particular, we find it interesting whether an arithmetic criterion similar to the $p$-large case (cf. Lemma 2) exists. Furthermore, in our reduction from VerTEX Cover, the construction yields a grid with a large amount of edges removed by taking a subgraph. Is MSE parametrised by the number of edges removed from the grid in FPT (or even admits a polynomial-size problem kernel)?

We consider it as interesting to study DMSE on Manhattan street networks (cf. [13]). Recently, MSE is considered with an additional time-aspect [14]. Herein, on a high level, an edge is shared if it appears in at least two paths at

\footnotetext{
${ }^{1} \mathrm{DMSE}$ is in FPT when parametrised by $p+k$ since the search tree algorithm solving MSE in $O\left((p-1)^{k} \cdot(|V|+|E|)^{2}\right)$ time [7] can easily be adapted to the directed case.
} 
the same time. Another future research direction could be to study MSE with the additional time-aspect on grid-like graphs.

\section{References}

[1] Samu Alanko, Simon Crevals, Anton Isopoussu, Patric R. J. Östergård, and Ville Pettersson. Computing the domination number of grid graphs. The Electronic Journal of Combinatorics, 18(1), 2011.

[2] Yusuke Aoki, Bjarni V. Halldórsson, Magnús M. Halldórsson, Takehiro Ito, Christian Konrad, and Xiao Zhou. The minimum vulnerability problem on specific graph classes. Journal of Combinatorial Optimization, 32(4):1288$1304,2016$.

[3] Sepehr Assadi, Ehsan Emamjomeh-Zadeh, Ashkan Norouzi-Fard, Sadra Yazdanbod, and Hamid Zarrabi-Zadeh. The minimum vulnerability problem. Algorithmica, 70(4):718-731, 2014.

[4] Dinesh Bhatia, Frank Thomson Leighton, Fillia Makedon, and Carolyn Haibt Norton. Improved algorithms for routing on two-dimensional grids. In Proc. of the 18th International Workshop on Graph-Theoretic Concepts in Computer Science (WG 1992), volume 657 of LNCS, pages 114-122. Springer, 1992.

[5] Hans L. Bodlaender, Bart M.P. Jansen, and Stefan Kratsch. Kernelization lower bounds by cross-composition. SIAM Journal on Discrete Mathematics, 28(1):277-305, 2014.

[6] Till Fluschnik. The parameterized complexity of finding paths with shared edges. Master's thesis, TU Berlin, March 2015.

[7] Till Fluschnik, Stefan Kratsch, Rolf Niedermeier, and Manuel Sorge. The parameterized complexity of the minimum shared edges problem. In Proc. of the 35th IARCS Annual Conference on Foundation of Software Technology and Theoretical Computer Science (FSTTCS 2015), volume 45 of LIPIcs, pages 448-462. Schloss Dagstuhl - Leibniz-Zentrum fuer Informatik, 2015.

[8] Till Fluschnik and Manuel Sorge. The minimum shared edges problem on planar graphs. arXiv preprint arXiv:1602.01385, 2016.

[9] M. R. Garey and David S. Johnson. Computers and Intractability: A Guide to the Theory of NP-Completeness. W. H. Freeman, 1979.

[10] Vít Jelínek. The rank-width of the square grid. Discrete Applied Mathematics, 158(7):841-850, 2010.

[11] Kanchana Kanchanasut. A shortest-path algorithm for Manhattan graphs. Information Processing Letters, 49(1):21-25, 1994.

[12] Mamadou Moustapha Kanté, Fatima Zahra Moataz, Benjamin Momège, and Nicolas Nisse. Finding paths in grids with forbidden transitions. In Proc. of the 41st International Workshop Graph-Theoretic Concepts 
in Computer Science (WG 2015), volume 9224 of LNCS, pages 154-168. Springer, 2015.

[13] NF Maxemchuk. Routing in the Manhattan street network. IEEE Transactions on Communications, 35(5):503-512, 1987.

[14] Marco Morik. The complexity of routing with collision avoidance. Bachelor's thesis, TU Berlin, June 2016.

[15] Masoud T. Omran, Jörg-Rüdiger Sack, and Hamid Zarrabi-Zadeh. Finding paths with minimum shared edges. Journal of Combinatorial Optimization, 26(4):709-722, 2013.

[16] Neil Robertson and Paul D. Seymour. Graph minors. III. Planar tree-width. Journal of Combinatorial Theory, Series B, 36(1):49-64, 1984.

[17] Emmanouel A. Varvarigos. Optimal communication algorithms for Manhattan street networks. Discrete Applied Mathematics, 83(1-3):303-326, 1998.

[18] Zhi-Qian Ye, Yi-Ming Li, Hui-Qiang Lu, and Xiao Zhou. Finding paths with minimum shared edges in graphs with bounded treewidth. In Proc. of the 9th International Conference on Foundations of Computer Science (FCS 2013), pages 40-46, 2013. 